\title{
Phytoprotection
}

\section{Élaboration de normes DRIS provisoires pour des transplants de céleri}

\author{
N. Tremblay, L.É. Parent et A. Gosselin
}

Volume 71, numéro 3, 1990

URI : https://id.erudit.org/iderudit/705994ar

DOI : https://doi.org/10.7202/705994ar

Aller au sommaire du numéro

\section{Éditeur(s)}

Société de protection des plantes du Québec (SPPQ)l

\section{ISSN}

0031-9511 (imprimé)

1710-1603 (numérique)

Découvrir la revue

Citer cet article

Tremblay, N., Parent, L.-É. \& Gosselin, A. (1990). Élaboration de normes DRIS provisoires pour des transplants de céleri. Phytoprotection, 71(3), 129-136. https://doi.org/10.7202/705994ar
Résumé de l'article

Nous avons utilisé une banque de données contenant 215 observations pour obtenir des normes DRIS (Diagnosis and Recommandation Integrated System) provisoires pour des transplants de céleri (Apium graveolens var. Dulce). La détermination des normes s'est faite en considérant un groupe de tête au rendement supérieur ou égal à $1600 \mathrm{~g} /$ plant ( $27 \%$ de la population). Sur 45 rapports nutritionnels mesurés dans la partie aérienne des transplants, 26 ont présenté des rapports de variance permettant de distinguer significativement le groupe produisant des rendements supérieurs. Le coefficient de corrélation entre l'indice de déséquilibre nutritionnel (IDN) et les rendements s'est révélé très significatif. Les normes provisoires ont été confrontées à un ensemble indépendant de données obtenues chez des producteurs de la région du sud de Montréal (Québec). Les IDN calculés sur ces plantes échantillonnées au stade implantation (environ 27 jours après la plantation) ont été significativement corrélés aux rendements, malgré le fait que le tissu échantillonné et le stade de croissance aient été relativement éloignés de ceux correspondant aux normes. Les normes ont permis d'identifier la cause probable d'un problème de croissance induit par l'utilisation d'une solution fertilisante ne convenant pas à la production de transplants.
Ce document est protégé par la loi sur le droit d'auteur. L'utilisation des services d'Érudit (y compris la reproduction) est assujettie à sa politique d'utilisation que vous pouvez consulter en ligne.

https://apropos.erudit.org/fr/usagers/politique-dutilisation/ 


\title{
Élaboration de normes DRIS provisoires pour des transplants de céleri
}

\author{
Nicolas Tremblay \\ Station de recherches, Agriculture Canada, 430, boul. Gouin \\ Saint-Jean-sur-Richelieu (Québec), Canada J3B 3E6. Contribution $N^{\circ} 335 / 90.10 .24 A R$
}

\section{Léon-Étienne Parent}

Département des sols, Faculté des sciences de l'agriculture et de l'alimentation, Université Laval, Québec (Québec), Canada GlK 7P4

\author{
André Gosselin \\ Département de phytologie, Faculté des sciences de l'agriculture et de l'alimentation, \\ Université Laval, Québec (Québec), Canada GlK 7P4
}

(Reçu 1989-11-03; accepté 1990-04-12)

\begin{abstract}
Nous avons utilisé une banque de données contenant 215 observations pour obtenir des normes DRIS (Diagnosis and Recommendation Integrated System) provisoires pour des transplants de céleri (Apium graveolens var. Dulce). La détermination des normes s'est faite en considérant un groupe de tête au rendement supérieur ou égal à $1600 \mathrm{~g} /$ plant $(27 \%$ de la population). Sur 45 rapports nutritionnels mesurés dans la partie aérienne des transplants, 26 ont présenté des rapports de variance permettant de distinguer significativement le groupe produisant des rendements supérieurs. Le coefficient de corrélation entre l'indice de déséquilibre nutritionnel (IDN) et les rendements s’est révélé très significatif. Les normes provisoires ont été confrontées à un ensemble indépendant de données obtenues chez des producteurs de la région du sud de Montréal (Québec). Les IDN calculés sur ces plantes échantillonnées au stade implantation (environ 27 jours après la plantation) ont été significativement corrélés aux rendements, malgré le fait que le tissu échantillonné et le stade de croissance aient été relativement éloignés de ceux correspondant aux normes. Les normes ont permis d'identifier la cause probable d'un problème de croissance induit par l'utilisation d'une solution fertilisante ne convenant pas à la production de transplants.
\end{abstract}

Tremblay, N., L.-É. Parent et A. Gosselin. 1990. Élaboration de normes DRIS provisoires pour des transplants de céleri. PHYTOPROTECTION 71: 129-136.

A data bank of 215 observations was used to elaborate preliminary DRIS norms for celery (Apium graveolens var. Dulce) transplants. The threshold yield for high yielders was set at $1600 \mathrm{~g} / \mathrm{plant}(27 \%$ of the population). Of the 45 nutrient ratios obtained, 26 presented significant variance ratios between low and high yielders. The correlation coefficient between nutrient imbalance index (NII) and yield was highly significant. The norms were tested on an independent set of data obtained from celery growers located south of Montreal (Québec). The NII obtained from plants at the implantation stage (about 27 days after transplanting) was significantly correlated to yield even if the sampled tissue was different from that used for norm calculations. The norms were also used to identify the likely cause of a growth problem induced by a nutrient solution not suited for transplant production.

\section{Introduction}

Depuis quelques années, l'industrie locale de transplants de légumes connaît un essor considérable. Pour rationaliser cette production, des expériences ont porté sur l'ajout de $\mathrm{CO}_{2}$ dans les serres, sur l'utilisation d'éclairage d'appoint et sur l'optimisation des fertilisations en $\mathrm{N}$ et $\mathrm{P}$ (Masson 1989; Tremblay et Gosselin 1989; Tremblay et al. 1987). Ces recherches ont mis en évidence le rôle déterminant d'une nutrition minérale équilibrée pour l'atteinte du potentiel de rendement chez les transplants de

$0031-9511 / 90 \$ 1.00+.10$ céleri (Apium graveolens L. var. Dulce). Or, seulement trois critères de valeur minimale critique $\left(\mathrm{NO}_{3}-\mathrm{N}, \mathrm{PO}_{4}-\mathrm{P}\right.$ et $\left.\mathrm{K}\right)$, pour deux stades de croissance au champ (misaison et pré-récolte), existent actuellement pour juger de l'équilibre nutritionnel dans le pétiole du céleri (Lorenz et Maynard 1980). L'absence de normes de référence pour les transplants de céleri en serre pose un problème aux producteurs désireux d'identifier les différents déséquilibres nutritionnels qui peuvent se manifester en cours de production et d'apporter dès que possible les correctifs qui s'imposent.

Le diagnostic des besoins en éléments fertilisants à partir des analyses foliaires est 
encore pour une large part basé sur le concept de la valeur minimale critique (VMC) définie comme la concentration de l'élément dans une certaine partie de la plante échantillonnée à un stade précis, sous laquelle le rendement potentiel baisse d'au moins 5 à $10 \%$. En pratique, cette approche rencontre de sérieuses contraintes (Sumner 1979).

Le DRIS (Diagnosis and Recommendation Integrated System) a été développé avec succès sur plusieurs cultures (Sumner 1979; Walworth et Sumner 1987) à partir des rapports d'éléments minéraux qui parviennent le mieux à caractériser les populations à hauts rendements (Beaufils 1973). Les rapports qui unissent les éléments entre eux sont souvent moins variables que les concentrations exprimées sur une base de matière sèche. Ainsi, les biais dus à l'âge du tissu, au cultivar et à la position du tissu échantillonné sur la plante sont considérablement diminués. Le DRIS résoudrait mieux les problèmes de déséquilibres nutritionnels que la VMC (Sumner 1979; Davee et al. 1986; Walworth et Sumner 1987).

Avec le DRIS, les rapports nutritionnels mesurés dans les tissus foliaires de plantes à forts rendements servent de référence permettant de quantifier dans quelle mesure un échantillon s'éloigne de la composition minérale d'une culture à rendement élevé (Letzsch 1985). Les plantes de référence obtenues dans un grand nombre de conditions de croissance (Letzsch et Sumner 1984; Walworth et al. 1988) sont utilisées à des fins diagnostiques. L'expression retenue du rapport nutritionnel $(\mathrm{x} / \mathrm{y}$ ou $\mathrm{y} / \mathrm{x})$ est celle correspondant au rapport le plus élevé entre la variance des plantes à faibles rendements (numérateur) et celle des plantes à rendements supérieurs (dénominateur). Au Québec, des normes DRIS ont été suggérées pour le pommier nain (Malus domestica Borkh.) (Parent et Granger 1989), le soja (Glycine max [L.] Merr.) (Vigier et al. 1989) et la tomate (Lycopersicon esculentum L.) (Caron et Parent 1989). La proposition de normes provisoires (Evanylo et al. 1987; Hockman et al. 1989; Savoy et Robinson 1989; Sumner 1977; Walworth et al. 1986) présente un intérêt certain puisque le gain de précision est de plus en plus faible à mesure que s'ajoutent les informations dans la banque de données (Letzsch et Sumner 1984).
Le calcul des indices DRIS permet de classer les éléments selon qu'ils causent une carence ou qu'ils sont en excès dans la plante. Les indices sont déterminés à partir de deux équations de calcul sur les distances pondérées (Walworth et Sumner 1987):

$f(x / y)=\{[(x / y) /(a / b)]-1\} k / C V$ si $x / y \geq a / b$

et

$f(\mathrm{x} / \mathrm{y})=[1-(\mathrm{a} / \mathrm{b}) /(\mathrm{x} / \mathrm{y})] \mathrm{k} / \mathrm{CV}$ si $x / y<a / b$

où $\mathrm{x} / \mathrm{y}$ est le rapport nutritionnel d'un échantillon indépendant, $\mathrm{a} / \mathrm{b}$ est la norme correspondante, $\mathrm{CV}$ est le coefficient de variation associé à $\mathrm{a} / \mathrm{b}$ et $\mathrm{k}$ est une constante fixée à 1000. L'utilisation des deux équations est nécessaire pour rendre le calcul des indices indépendant de l'expression du rapport nutritionnel; la relation $f(\mathrm{x} / \mathrm{y})=$ $-f(\mathrm{y} / \mathrm{x})$ doit être respectée. L'indice nutritionnel est la moyenne de toutes les fonctions DRIS dans lesquelles un élément particulier est impliqué; si l'élément est situé au numérateur, la fonction est multipliée par +1 et, s'il se situe au dénominateur, la fonction est multipliée par -1 .

L'indice du déséquilibre nutritionnel (IDN) est la somme des indices transformés en valeurs absolues. Les plantes montrant des IDN élevés sont moins susceptibles de produire de hauts rendements que celles à IDN faibles; l'IDN présente généralement une corrélation inverse avec le rendement (Walworth et Sumner 1987).

L'objectif de cette recherche est donc de développer des normes DRIS provisoires chez les transplants de céleri et d'évaluer leur valeur pour pronostiquer le rendement d'échantillons indépendants.

\section{Matériel et méthodes}

Procédures de calcul des normes provisoires. Les données utilisées pour les fins de calcul des normes provisoires sont tirées de Tremblay et al. (1987, 1988), Tremblay et Gosselin (1989), Masson (1989) et Tremblay et al. (données non publiées). Dans le cadre de ces recherches, les plants ayant reçu divers traitements expérimentaux 
sont évalués ai moment de la plantation pour leurs caractéristiques végétatives. Un échantillon des parties aériennes complètes est ensuite séché et soumis aux procédures d'analyses minérales. Entre huit et dix plants par unité expérimentale sont sélectionnés au hasard parmi ceux restant et sont introduits en culture commerciale sur un sol organique chez un producteur de la région de Napierville (Québec). La récolte se fait au moment où les plantes sont considérées vendables, soit après une accumulation de degrés-jours (base $7^{\circ} \mathrm{C}$ ) variant entre 745 et 955. Pour uniformiser les données de productivité d'une année à l'autre, tous les rendements dont il est question dans cet article sont ajustés en postulant un gain de poids proportionnel à une accumulation hypothétique de 1000 degrés-jours de croissance au champ, tel que mesuré à la ferme expérimentale d'Agriculture Canada à SainteClotilde.

L'élaboration des normes est inspirée des procédures décrites par Walworth et Sumner (1987), en considérant un groupe de rendements supérieurs ou égaux à $1600 \mathrm{~g} /$ plant qui représente $27 \%$ de 215 observations. Les rapports nutritionnels sont calculés sur toutes les combinaisons possibles entre les variables suivantes, exprimées sur une base de matière sèche: $\mathrm{N}$, $\mathrm{P}, \mathrm{K}, \mathrm{Ca}, \mathrm{Mg}(\%)$, ainsi que $\mathrm{B}, \mathrm{Fe}, \mathrm{Mn}$ et $\mathrm{Zn}$ (ppm).

\section{Évaluation de la qualité pronostique} des normes provisoires. La valeur pronostique des normes est évaluée sur un premier ensemble d'échantillons indépendants recueillis chez des producteurs de céleri pendant la saison 1987. Cet échantillonnage de 56 parcelles est effectué sur divers types de sols organiques et modes de régie chez cinq producteurs de la région du sud de Montréal. Les dates de plantation varient entre le 4 mai et le 2 juillet. Des échantillons sont recueillis au hasard dans chaque parcelle en moyenne 27 jours après la plantation. Les échantillons sont constitués des limbes et des pétioles de feuilles jeunes et matures. Les rendements, mesurés entre le 19 juillet el le 5 octobre, sont estimés sur la partie aérienne complète de sept plantes obtenues au hasard dans chaque parcelle, puis pondérés sur la base de 1000 degrés- jours de croissance à partir de la date de plantation.

Un second ensemble de données sert à estimer la valeur pronostique des normes provisoires pendant la saison 1989. Dans le cadre de cette expérience, quatre solutions fertilisantes sont comparées quant à leur effet sur la croissance de transplants et leurs conséquences à long terme sur les rendements. Le semis est effectué le 31 mars 1989 dans des plateaux alvéolés de polystyrène (Speedling Inc.) contenant un substrat à base de tourbe de sphaigne (Metro Mix 200, W.R. Grace \& Co. du Canada Ltée, Ajax, Ontario). Le terreau est maintenu humide avec de l'eau du robinet de $\mathrm{pH} 6,5$ et de conductivité électrique $0,2 \mathrm{mS} / \mathrm{cm}$. Chaque unité expérimentale est constituée d'une partie de plateau « Speedling » modèle 080A $\left(8,5 \mathrm{~cm}^{3} /\right.$ alvéole $)$. Les plants sont disposés dans une serre de verre à la Station de recherches d'Agriculture Canada à SaintJean-sur-Richelieu, sous un éclairage d'appoint fourni par des lampes à haute pression de sodium (HPS) d'environ 50 $\mu \mathrm{mol} /\left(\mathrm{m}^{2} \cdot \mathrm{s}\right)$ (PAR). La période d'utilisation de l'éclairage d'appoint est de $16 \mathrm{~h}$ (06:00 à 22:00) sauf lors de périodes sans nuages alors que l'éclairage est interrompu jusqu'à 16:00. Les traitements de fertilisation débutent au semis et sont appliqués matin et soir au moyen d'un arrosoir jusqu'au drainage partiel des cellules.

Trois solutions fertilisantes commerciales et une autre préparée en laboratoire constituent les traitements expérimentaux. La solution 1 est préparée à partir de l'engrais soluble Peters ${ }^{\circledR}$ 20-19-18 (20N-8,3P-15K) Peat-lite special. La solution 2 vient du Peters $^{\circledR}$ 20-5-30 (20N-2,2P-25K) Potash special. La solution 3 vient du Peters ${ }^{\circledR}$ 20-10-20 (20N-4,4P-16,6K) Peat-lite special. Les solutions commerciales sont préparées avec de l'eau du robinet. Finalement, la solution 4, préparée en laboratoire avec de l'eau déminéralisée, représente un engrais 20-20-8 (20N-8,6P-6,8K). Toutes les solutions contiennent aussi les éléments suivants: $\mathrm{Mg}, \mathrm{Fe}, \mathrm{Mn}, \mathrm{B}, \mathrm{Zn}, \mathrm{Cu}$ et Mo. Le $\mathrm{pH}$ des solutions commerciales n'est pas ajusté. Le $\mathrm{pH}$ de la solution 4 est ajusté à 5,5 avec du $\mathrm{NaOH}$. Sa composition détaillée, en ppm, est la suivante: $350 \mathrm{~N}$ (rapport 2:1:9 de $\mathrm{N}-\mathrm{NO}_{3}: \mathrm{N}-\mathrm{NH}_{4}: \mathrm{N}$-Urée), $150 \mathrm{P}, 119 \mathrm{~K}$, 
$50 \mathrm{Ca}, 20 \mathrm{Mg}, 2 \mathrm{Fe}$ (sous la forme du chélat DTPA), 0,5 Mn, 0,03 Cu, 0,02 Mo et 0,05 $\mathrm{Zn}$. À partir du $17^{\mathrm{e}}$ jour, les solutions sont appliquées matin et soir à raison de 100 ppm N. A partir du $21^{\mathrm{e}}$ jour, les solutions sont appliquées à raison de $150 \mathrm{ppm} \mathrm{N}$ et par la suite la concentration de la solution augmente de 50 ppm $\mathrm{N}$ à tous les 7 jours.

Avant l'échantillonnage, la partie foliaire est rincée par une application d'eau distillée. Le poids de la matière sèche des parties aérienne et racinaire est mesuré 53 jours après le semis alors que des échantillons de 16 plants sont prélevés hors des bordures du plateau. La partie aérienne est sectionnée au niveau du substrat.

Les plants restants sont plantés le lendemain, 24 mai 1989, à deux emplacements à raison de six blocs complets par emplacement. Le premier est le champ du producteur en sols organiques de Napierville où la parcelle expérimentale fait partie intégrante des plantes exploitées commercialement. Le second emplacement est à la ferme expérimentale d'Agriculture Canada à SainteClotilde où les plantes sont seulement l'objet d'opérations culturales commerciales. La récolte est faite le 11 août 1989 , après 79 jours de croissance au champ. La partie aérienne complète de quatre plantes par unité expérimentale est alors pesée.

Analyse minérale. Tous les échantillons sont séchés au four à $70^{\circ} \mathrm{C}$ puis broyés et digérés selon la méthode de Isaac et Johnson (1976). La teneur en $\mathrm{N}$ et en $\mathrm{P}$ est déterminée par la méthode Technicon AutoAnalyser II-C (No. 334-74 W/B+) et les concentrations en $\mathrm{K}, \mathrm{Ca}, \mathrm{Mg}, \mathrm{B}, \mathrm{Cu}, \mathrm{Fe}$, Mn et $\mathrm{Zn}$ par spectroscopie d'émission atomique à plasma.

\section{Résultats et discussion}

Normes provisoires. Des 45 rapports nutritionnels sélectionnés, 26 ont présenté des rapports de variances permettant de caractériser significativement le groupe des plantes à rendements élevés (tableau 1). Le rapport $\mathrm{MS} / \mathrm{Ca}$ (matière sèche $/ \mathrm{Ca}$; dont l'inverse est utilisé dans la méthode VMC) n'est pas significatif mais cinq rapports impliquant le $\mathrm{Ca}$ le sont. Le coeur noir du céleri pourrait donc être mieux diagnostiqué par le DRIS que par la VMC. Également, la plupart des rapports incluant le $\mathrm{Mg}$, le $\mathrm{Fe}$ ou le $\mathrm{Zn}$ revêtent une valeur significative. Bien que 19 rapports ne soient pas significatifs, leur présence est nécessaire dans les opérations de calcul, leur valeur étant pondérée en conséquence par les procédures de calcul des indices.

La figure 1 représente la relation entre l'IDN et les rendements dans le cas des transplants de céleri utilisés pour le calcul des normes provisoires. Le coefficient de corrélation est très significatif ( $r=-0,48$; $P \leq 0,001)$, et la relation indique que des rendements élevés sont associés aux IDN se rapprochant de zéro.

Évaluation de la qualité pronostique des normes provisoires. La validation des normes est généralement faite au moyen d'une expérience de type factoriel où l'impact sur les rendements des corrections suggérées par le DRIS est interprété en terme de succès ou d'échec (Walworth et Sumner 1987). Des normes ont déjà été proposées sans qu'aucune validation ne soit tentée (Hockman et al. 1989). Une façon alternative de témoigner de la qualité de normes provisoires serait de mettre en relation l'IDN et les rendements en utilisant des ensembles indépendants de données. À cette fin, des indices DRIS ont été obtenus à partir de l'analyse minérale des échantillons recueillis chez les producteurs au cours de la saison 1987.

La figure 2 présente la relation graphique entre les rendements totaux et les IDN estimés. La relation inverse significative constitue une indication à l'effet que les normes provisoires développées pour les transplants permettent de juger de l'équilibre nutritionnel de jeunes plantes. Cette observation va dans le sens des travaux qui soulignent l'importance d'un conditionnement nutritionnel pré-plantation (CNP) adapté à la préparation des transplants de céleri (Dufault 1986; Masson 1989; Tremblay et al. 1987, 1988). À la lumière de nos résultats, l'objectif du CNP devrait être dans une large mesure d'obtenir des plants dont la composition minérale se rapproche d'un optimum théorique représenté par les normes DRIS. Selon les normes provisoires, les carences les plus fréquemment rencontrées chez les 
Tableau 1. Normes DRIS provisoires pour des transplants de céleri

\begin{tabular}{|c|c|c|c|c|c|c|c|}
\hline \multirow[b]{2}{*}{$\begin{array}{l}\text { Rapport } \\
\text { nutritif }\end{array}$} & \multicolumn{3}{|c|}{$\begin{array}{c}\text { Population à } \\
\text { faibles rendements } \\
(\mathrm{n}=156)\end{array}$} & \multicolumn{3}{|c|}{$\begin{array}{l}\text { Population à } \\
\text { hauts rendements } \\
(\mathrm{n}=59)\end{array}$} & \multirow[b]{2}{*}{$\mathrm{S}_{\mathrm{A}} / \mathrm{S}_{\mathrm{B}}$} \\
\hline & Moyenne & $\begin{array}{l}\mathrm{CV} \\
(\%) \\
\end{array}$ & $\begin{array}{c}\text { Variance } \\
\left(\mathrm{S}_{\mathrm{A}}\right)^{\S} \\
\end{array}$ & Moyenne & $\begin{array}{l}\mathrm{CV} \\
(\%)\end{array}$ & $\begin{array}{c}\text { Variance } \\
\left(\mathrm{S}_{\mathrm{B}}\right)\end{array}$ & \\
\hline$\overline{\mathrm{N} / \mathrm{MS}^{\dagger}}$ & 3,735 & 28,8 & 1,16 & 2,884 & 30,2 & 0,76 & $1,53^{*}$ \\
\hline $\mathrm{N} / \mathrm{P}$ & 3,189 & 31,4 & 1,00 & 1,912 & 50,5 & 0,93 & 1,08 \\
\hline $\mathrm{N} / \mathrm{K}$ & 0.846 & 39,4 & 0,11 & 0,605 & 35,0 & 0,04 & $2,48 * *$ \\
\hline $\mathrm{N} / \mathrm{Ca}$ & 3,086 & 32,1 & 0,98 & 2,586 & 47,0 & 1,48 & 0,67 \\
\hline $\mathrm{N} / \mathrm{Mg}$ & 7,359 & 28,9 & 4,51 & 7,723 & 38,4 & 8,80 & 0,51 \\
\hline $\mathrm{N} / \mathrm{B}$ & 0,075 & 56,7 & $1,8 \times 10^{-3}$ & 0,042 & 43,4 & $3,3 \times 10^{-4}$ & $5,43 * *$ \\
\hline $\mathrm{N} / \mathrm{Fe}$ & 0,020 & 39,7 & $6,5 \times 10^{-5}$ & 0,019 & 39,2 & $5,4 \times 10^{-5}$ & 1,21 \\
\hline $\mathrm{N} / \mathrm{Mn}$ & 0,054 & 42,1 & $5,2 \times 10^{-4}$ & 0,054 & 55,3 & $8,8 \times 10^{-4}$ & 0,59 \\
\hline $\mathrm{K} / \mathrm{P}$ & 4,129 & 38,1 & 2,47 & 3,365 & 53,0 & 3,18 & 0,78 \\
\hline $\mathrm{K} / \mathrm{Ca}$ & 4,009 & 36,1 & 2,10 & 4,652 & 46,1 & 4,59 & 0,46 \\
\hline $\mathrm{K} / \mathrm{Mn}$ & 0,080 & 65,7 & $2,8 \times 10^{-3}$ & 0,095 & 51,2 & $2,3 \times 10^{-3}$ & 1,18 \\
\hline $\mathrm{Ca} / \mathrm{P}$ & 1,070 & 26,8 & 0,08 & 0,806 & 39,4 & 0,10 & 0,81 \\
\hline $\mathrm{Ca} / \mathrm{B}$ & 0,246 & 49,8 & $1,5 \times 10^{-4}$ & 0,019 & 43,9 & $7,3 \times 10^{-5}$ & $2,07 * *$ \\
\hline $\mathrm{Mg} / \mathrm{MS}$ & $0,528^{\circ}$ & 30,3 & 0,03 & 0,391 & 18,5 & $5,2 \times 10^{-3}$ & $4,92 * *$ \\
\hline $\mathrm{Mg} / \mathrm{P}$ & 0,453 & 35,0 & 0,03 & 0,248 & 32,5 & $6,5 \times 10^{-3}$ & $3,85^{* *}$ \\
\hline $\mathrm{Mg} / \mathrm{K}$ & 0,121 & 42,3 & 0,03 & 0,086 & 44,9 & $1,5 \times 10^{-3}$ & $1,76 * *$ \\
\hline $\mathrm{Mg} / \mathrm{Ca}$ & 0,423 & 24,3 & 0,01 & 0,328 & 23,0 & $5,7 \times 10^{-3}$ & $1,87 * *$ \\
\hline $\mathrm{Mg} / \mathrm{B}$ & 0,011 & 58,6 & $4,1 \times 10^{-5}$ & 0,006 & 40,4 & $5,9 \times 10^{-6}$ & $6,94 * *$ \\
\hline $\mathrm{Mg} / \mathrm{Fe}$ & 0,003 & 34,3 & $9,0 \times 10^{-7}$ & 0,003 & 29,4 & $5,0 \times 10^{-7}$ & $1,80^{* *}$ \\
\hline $\mathrm{Mg} / \mathrm{Mn}$ & 0,007 & 29,2 & $4,6 \times 10^{-6}$ & 0,007 & 32,2 & $4,7 \times 10^{-6}$ & 0,98 \\
\hline $\mathrm{Mg} / \mathrm{Zn}$ & 0,015 & 47,3 & $5,2 \times 10^{-5}$ & 0,017 & 50,3 & $7,2 \times 10^{-5}$ & 0,73 \\
\hline $\mathrm{B} / \mathrm{P}$ & 56,824 & 58,5 & 1106,79 & 54,998 & 70,0 & 1480,84 & 0,75 \\
\hline $\mathrm{B} / \mathrm{K}$ & 14,404 & 60,5 & 76,04 & 17,964 & 63,4 & 129,82 & 0,59 \\
\hline $\mathrm{Fe} / \mathrm{MS}$ & 206,635 & 41,7 & 7408,33 & 169,129 & 34,7 & 3443,89 & $2,15^{* *}$ \\
\hline $\mathrm{Fe} / \mathrm{P}$ & 175,992 & 45,2 & 6320,77 & 8,077 & 42,3 & 2093,00 & $3,02 * *$ \\
\hline $\mathrm{Fe} / \mathrm{K}$ & 47,001 & 53,2 & 625,48 & 37,475 & 54,3 & 414,26 & $1,51^{*}$ \\
\hline $\mathrm{Fe} / \mathrm{Ca}$ & 168,644 & 44,4 & 5600,13 & 141,679 & 39,2 & 3079,32 & $1,82 * *$ \\
\hline $\mathrm{Fe} / \mathrm{B}$ & 4.061 & 61,0 & 6,14 & 2,542 & 53,0 & 1,82 & $3,38 * *$ \\
\hline $\mathrm{Mn} / \mathrm{MS}$ & 81,808 & 47,0 & 1479,08 & 66,349 & 45,6 & 915,40 & $1,62 *$ \\
\hline $\mathrm{Mn} / \mathrm{P}$ & 68,322 & 48,2 & 1084,25 & 38,865 & 31,5 & 149,76 & $7,24 * *$ \\
\hline $\mathrm{Mn} / \mathrm{Ca}$ & 64,564 & 44,3 & 816,93 & 51,024 & 21,9 & 125,04 & $6,53^{* *}$ \\
\hline $\mathrm{Mn} / \mathrm{B}$ & 1,734 & 66,2 & 1,32 & 1,025 & 50,4 & 0,27 & $4,94 * *$ \\
\hline $\mathrm{Mn} / \mathrm{Fe}$ & 0,427 & 51,5 & 0,05 & 0,413 & 41,4 & 0,03 & $1,66^{* *}$ \\
\hline $\mathrm{Zn} / \mathrm{MS}$ & 42,575 & 54,0 & 528,65 & 29,785 & 50,6 & 226,77 & $2,33^{* *}$ \\
\hline $\mathrm{Zn} / \mathrm{N}$ & 11,452 & 53,3 & 37,29 & 10,667 & 54,0 & 33,20 & 1,12 \\
\hline $\mathrm{Zn} / \mathrm{P}$ & 35,162 & 57,0 & 401,04 & 18,306 & 53,4 & 95,44 & $4,20 * *$ \\
\hline $\mathrm{Zn} / \mathrm{K}$ & 9,977 & 66,3 & 43,77 & 6,817 & 71,7 & 23,89 & $1,83^{* *}$ \\
\hline $\mathrm{Zn} / \mathrm{Ca}$ & 33,648 & 53,7 & 326,17 & 23,489 & 48,8 & 131,58 & $2,48^{* *}$ \\
\hline $\mathrm{Zn} / \mathrm{B}$ & 0,851 & 74,0 & 0,40 & 0,399 & 46,1 & 0,03 & $11,73^{* *}$ \\
\hline $\mathrm{Zn} / \mathrm{Fe}$ & 0,217 & 49,2 & 0,01 & 0,180 & 42,7 & 0,01 & $1,93 * *$ \\
\hline $\mathrm{Zn} / \mathrm{Mn}$ & 0,557 & 49,8 & 0,08 & 0,493 & 61,0 & 0,09 & 0,85 \\
\hline $\mathrm{MS} / \mathrm{P}$ & 87,107 & 27,3 & 563,73 & 65,814 & 41,3 & 740,07 & 0,76 \\
\hline $\mathrm{MS} / \mathrm{K}$ & 22,237 & 20,9 & 21,66 & 21,312 & 26,7 & 32,37 & 0,67 \\
\hline $\mathrm{MS} / \mathrm{Ca}$ & 84,046 & 24,3 & 418,49 & 88,473 & 33,2 & 862,55 & 0,49 \\
\hline $\mathrm{MS} / \mathrm{B}$ & 1,908 & 38,5 & 0,54 & 1,575 & 45,1 & 0,50 & 1,07 \\
\hline
\end{tabular}

$\S S_{A}=$ variance du rapport nutritif de la population à faibles rendements; $S_{B}=$ variance du rapport nutritif de la population à hauts rendements.

$\dagger \mathrm{MS}=$ masse de la matière sèche.

* Significatif à $P \leq 0,05 ; * *$, significatif à $P \leq 0,01$. 


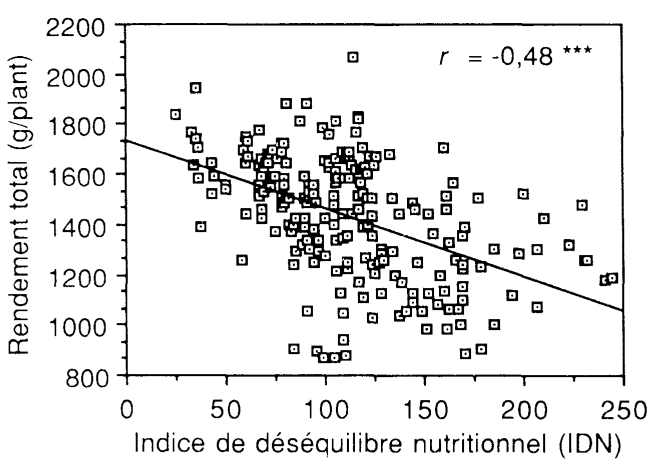

Figure 1. Relation entre l'indice de déséquilibre nutritionnel (IDN) et les rendements du céleri chez les plants ayant fait l'objet du calcul des normes. ***, coefficient de corrélation linéaire significatif à $0,1 \%$.

plantes des producteurs en 1987 sont: $\mathrm{N}>>\mathrm{Mg}=\mathrm{Mn}>\mathrm{K}=\mathrm{Fe}=\mathrm{Zn}$. Les excès les plus fréquents sont: $\mathrm{Fe}>>\mathrm{Ca}>$ $\mathrm{P}>\mathrm{Mn}$.

Le seuil de $1600 \mathrm{~g} /$ plant regroupe $27 \%$ des observations dans le groupe de tête pour les plants ayant fait l'objet du calcul des normes mais il en regroupe $63 \%$ pour les plantes des producteurs, ce qui est nettement excessif. L'éventuel enrichissement de la base de données devrait aller dans le sens de la sélection d'un seuil plus élevé (Letzsch et Sumner 1984) qui serait mieux adapté à la réalité des producteurs.

La solution 2 a réduit le développement de la partie aérienne des transplants sans affecter pour autant la partie racinaire (tableau 2 ). Une carence en $\mathrm{Zn}$ pourrait bien être la

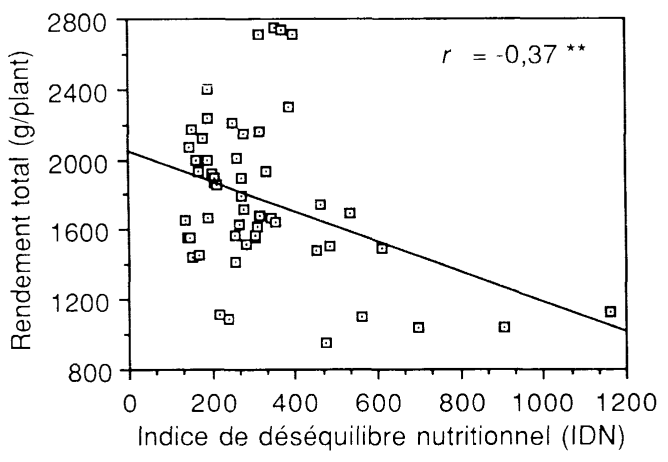

Figure 2. Relation entre l'indice de déséquilibre nutritionnel (IDN) des plants des producteurs au stade implantation et les rendements du céleri en 1987. **, coefficient de corrélation linéaire significatif à $1 \%$.

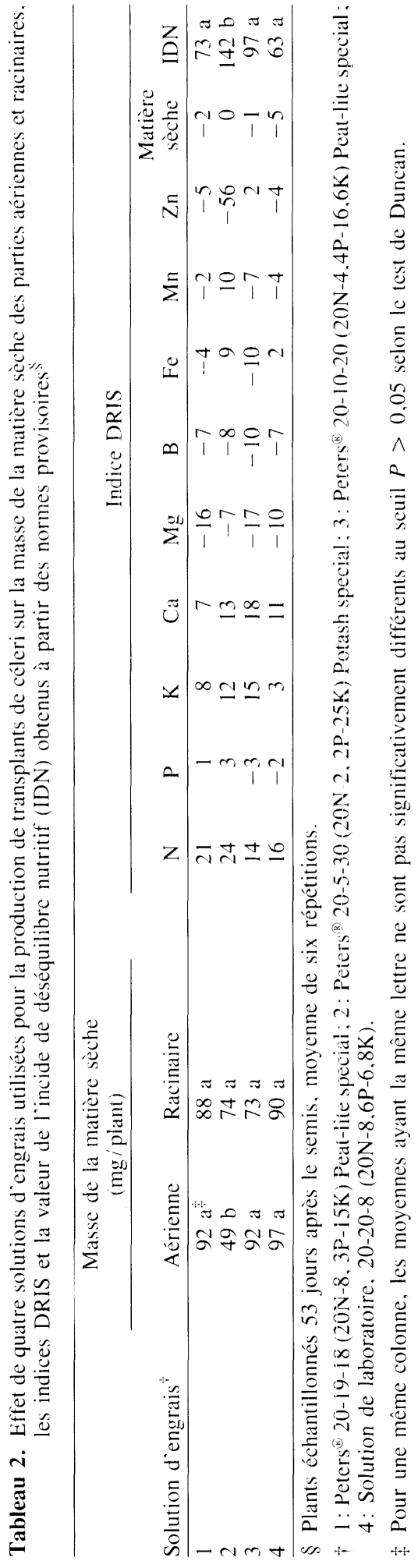


cause des problèmes associés à l'utilisation de la solution 2. Cette dernière fournit 0,04 ppm Zn (à la dilution de 350 ppm N), ce qui représente $14 \%$ du $\mathrm{Zn}$ des solutions 1 et 3 , et $88 \%$ du $\mathrm{Zn}$ de la solution 4 . Il faut envisager également la possibilité que le manque relatif de $\mathrm{P}$ dans la solution 2 (Peters ${ }^{\circledR}$ 20-5-30 [20N-2,2P-25K] Potash special) ait indirectement altéré le contenu en $\mathrm{Zn}$ des plants par l'intermédiaire d'une interaction P-Zn (Singh et al. 1988).

Les plants de tous les traitements ont connu un excès de $\mathrm{N}, \mathrm{K}$ et de $\mathrm{Ca}$ mais un déficit de $\mathrm{Mg}$ et $\mathrm{B}$ (tableau 2). En appliquant le DRIS à la préparation des transplants, on peut envisager non seulement de corriger les carences mais aussi d'atténuer les excès nutritionnels grâce à la souplesse que permet l'utilisation de solutions hydroponiques. En champ, l'intervention consécutive au diagnostic se limite souvent à l'ajout de l'élément le plus susceptible de causer une carence.

La différence significative $(P \leq 0,05)$ de développement de la partie aérienne des plants du traitement 2 se traduit dans la valeur de l'IDN (tableau 2). Autrement, les valeurs des IDN sont comparables et relativement faibles. Sur cette base, on peut prévoir que les rendements ne devraient pas être limités par l'équilibre nutritionnel des transplants. On n'a effectivement relevé aucune influence significative de la solution fertilisante sur les rendements. La récolte obtenue des plants préparés avec la solution 2 n'a toutefois représenté que $90 \%$ de celle des plants des trois autres traitements.

L'intérêt des normes provisoires semble être fonction des conditions particulières vécues par les transplants pendant leur croissance au champ. De meilleurs rendements ont été obtenus en moyenne à Napierville (1614 g/plant; fig. 3A) qu'à Sainte-Clotilde (1294 g/plant; fig. 3B). Les conditions de croissance ont donc été moins limitantes au premier emplacement puisque les transplants avaient la même origine. C'est pourquoi la relation qui se manifeste entre l'IDN et le rendement s'est également mieux exprimée (bien que non-significativement) à Napierville par rapport à SainteClotilde. Cette observation suggère que le potentiel de rendement que représente
A)

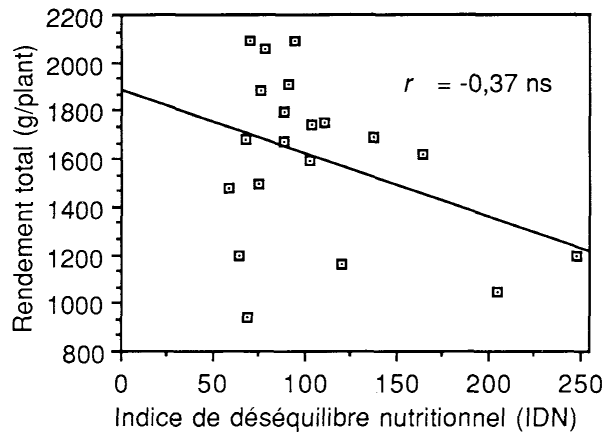

B)

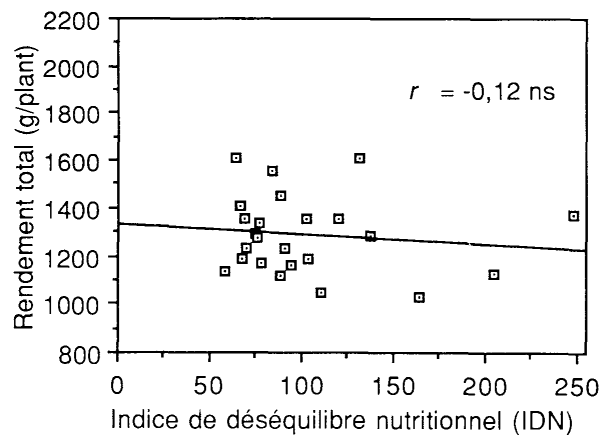

Figure 3. Relation entre l'indice de déséquilibre nutritionnel (IDN) de plants préparés avec quatre solutions fertilisantes et les rendements du céleri à deux emplacements de plantation en 1989: A) Napierville; B) Sainte-Clotilde. NS, coefficient de corrélation linéaire non-significatif.

l'IDN n'est significatif que si les conditions prévalant au champ ne limitent pas sa pleine expression.

\section{Conclusion}

Nos résultats mettent en perspective pour la première fois l'importance de l'équilibre nutritionnel dans l'expression du rendement même chez de très jeunes transplants maraîchers. Les normes provisoires retenues ont une valeur pronostique du potentiel de rendement de transplants de céleri aux champs depuis environ 27 jours. Les normes aident aussi à expliquer les retards de croissance des transplants en serre. Dans le cas de la culture de transplants, on peut même envisager la correction des excès aussi bien que des carences nutritionnels. La banque de données ayant servi aux calculs présentés compte 215 observations. Ce nombre peut convenir à l'énoncé de normes provisoires 
mais il est nécessaire d'améliorer la précision de nos estimations avec d'autres données afin d'établir des normes DRIS pour les transplants de céleri. Toutefois, l'obtention d'une culture de transplants à la composition nutritionnelle équilibrée n'est un objectif valable que dans la mesure où les conditions de croissances subséquentes ne sont pas limitantes.

Les auteurs désirent remercier messieurs Yvon Perron, Jean Masson, Guy Beaudoin et Benoît Martel pour leur aide technique ainsi que les producteurs qui ont collaboré à l'échantillonnage en 1987.

Beaufils, E.R. 1973. Diagnosis and recommendation integrated system (DRIS). Univ. of Natal Soil Sci. Bull. 1. $130 \mathrm{pp}$.

Caron, J. et L.-É. Parent. 1989. Derivation and assessment of DRIS norms for greenhouse tomatoes. Can. J. Plant Sci. 69: 1027-1035.

Davee, D.E., T.L. Righetti, E. Fallahi et S. Robbins. 1986. An evaluation of the DRIS approach for identifying mineral limitations on yield in 'Napolean' sweet cherry. J. Am. Soc. Hortic. Sci. 111: 988-993.

Dufault R.J. 1986. Influence of nutritional conditioning on muskmelon transplant quality and early yield. J. Am. Soc. Hortic. Sci. 111: 698-703.

Evanylo, G.K., M.E. Sumner et W.S. Letzsch. 1987. Preliminary development and testing of DRIS soil norms for soybean production. Commun. Soil Sci. Plant Anal. 18: 1355-1377.

Hockman, J.N., J.A. Burger et D.W. Smith. 1989. A DRIS application to Fraser fir Christmas trees. Commun. Soil Sci. Plant Anal. 20: 305-318.

Isaac, R.A. et W.C. Johnson. 1976. Determination of total nitrogen in plant tissue using a block digestor. J. Assoc. Off. Anal. Chem. 59: 98-100.

Letzsch, W.S. 1985. Computer program for selection of norms for use in the diagnosis and recommendation integrated system. Commun. Soil Sci. Plant Anal. 16: 339-347

Letzsch, W.S. et M.E. Sumner. 1984. Effect of population size and yield level in selection of diagnosis and recommendation integrated system (DRIS) norms. Commun. Soil Sci. Plant Anal. 15 : 997-1006.
Lorenz, O.A. et D.N. Maynard. 1980. Knott's handbook for vegetable growers. $2^{\mathrm{c}}$ éd. John Wiley \& Sons, New York. 390 pp.

Masson, J. 1989. Influence de l'éclairage d'appoint et de la fertilisation azotée sur la croissance, la nutrition minérale et la productivité des transplants de légumes cultivés en plateaux multicellulaires. Thèse de maittrise. Université Laval, Québec. 136 pp.

Parent, L.-É. et R.L. Granger. 1989. Derivation of DRIS norms from a high density apple orchard established in the Québec Appalachians. J. Am. Soc. Hortic. Sci. 114: 915-919.

Savoy, H.J. et D.L. Robinson. 1989. Development and evaluation of preliminary DRIS norms for Dallisgrass. Commun. Soil Sci. Plant Anal. 20: 655-683.

Singh, J.P., R.E. Karamanos et J.W.B. Stewart. 1988. The mechanism of phosphorus-induced zinc deficiency in bean (Phaseolus vulgaris L.). Can. J. Soil Sci. 68: 345-358.

Sumner, M.E. 1977. Preliminary N, P, and K foliar diagnostic norms for soybeans. Agron. J. 69: 226-230.

Sumner, M.E. 1979. Interpretation of foliar analyses for diagnostic purposes. Agron. J. 71: 343-348.

Tremblay, N. et A. Gosselin. 1989. Growth, nutrient status, and yield of celery seedlings in response to urea fertilization. HortScience 24: 288-291.

Tremblay, N., S. Yelle et A. Gosselin. 1987. Effects of $\mathrm{CO}_{2}$ enrichment, nitrogen and phosphorus fertilization on growth and yield of celery transplants. HortScience 22: 875-876.

Tremblay, N., S. Yelle et A. Gosselin. 1988. Effects of $\mathrm{CO}_{2}$ enrichment, nitrogen and phosphorus fertilization during the nursery period on mineral composition of celery. J. Plant Nutr. 11: 37-49.

Vigier, B., A.F. Mackenzie et Z. Chen. 1989. Evaluation of diagnosis and recommendation integrated system (DRIS) on early maturing soybeans. Commun. Soil Sci. Plant Anal. 20: 685-693.

Walworth, J.L. et M.E. Sumner. 1987. The diagnosis and recommendation integrated system (DRIS). Adv. Soil Sci. 6: 149-188.

Walworth, J.I., M.E. Sumner, R.A. Isaac et C.O. Plank. 1986. Preliminary DRIS norms for alfalfa in the Southeastern United States and a comparison with Midwestern norms. Agron. J. 78: 1046-1052.

Walworth, J.L., H.J. Woodard et M.E. Sumner. 1988. Generation of corn tissue norms from a small, high-yield data base. Commun. Soil Sci. Plant Anal. 19: 563-577. 